\title{
AN INSERTION STRATEGY FOR A TWO-DIMENSIONAL SPATIAL ACCESS METHOD
}

\author{
Wendy Osborn \\ Department of Mathematics and Computer Science, University of Lethbridge, Lethbridge, Alberta, Canada, T1K $3 M 4$ \\ wendy.osborn@uleth.ca \\ Ken Barker \\ Department of Computer Science, University of Calgary, Calgary, Alberta, Canada, T2N 1N4 \\ barker@cpsc.ucalgary.ca
}

Keywords: $\quad$ Spatial access methods, multidimensional, hierarchical, data structures, performance.

\begin{abstract}
This paper presents the 2DR-tree, a novel approach for accessing spatial data. The 2DR-tree uses nodes that are the same dimensionality as the data space. All spatial relationships between objects are preserved. A validity rule ensures that every node preserves the spatial relationships among its objects. The proposed insertion strategy adds a new object by recursively partitioning the space occupied by a set of objects. A performance evaluation shows the advantages of the 2DR-tree and identifies issues for future consideration.
\end{abstract}

\section{INTRODUCTION}

A spatial database contains a large collection of objects that are located in multidimensional space. An important research issue in spatial databases is the efficient retrieval of objects based on location, using spatial access methods (SAMs).

No n-dimensional to one-dimensional mapping of spatial data exists that preserves all spatial relationships between objects (Gaede and Günther, 1998). However, SAMs use approaches that are intended for alphanumeric data. This leads to a one-dimensional spatial organization of objects. This also leads to unnecessary searching within a node and the data structure as a whole, because the only option is a linear search of a node in its entirety. It is not possible to search only part of a node.

This paper describes a portion of our work on a new hierarchical SAM to alleviate this limitation. The 2DR-tree fits the existing object space by using nodes of the same dimensionality. Therefore, twodimensional nodes are used to index objects in twodimensional space. Objects in each node are organized using a validity rule that preserves all spatial relationships. This strategy allows the 2DR-tree to support non-linear searching strategies. We expect that this strategy will reduce the amount of searching that is performed in a node. In addition, overcoverage and overlap will also be reduced.

\section{RELATED WORK}

Many approaches for indexing objects based on location are proposed in the literature (see (Gaede and Günther, 1998; Samet, 1990; Shekhar and Chawla, 2003) for surveys). These approaches are classified into three categories (Gaede and Günther, 1998): main memory methods, point access methods and spatial access methods (SAMs). Many important strategies are proposed in all categories. We focus on SAMs, since our work is in this category.

SAMs provide uniform access to both point and object data. Also, they remain height-balanced in the presence of a dynamic object set. Many SAMs are proposed in the literature (Guttman, 1984; Beckmann et al., 1990; Berchtold et al., 1996; Kamel and Faloutsos, 1994; Sellis et al., 1987; Orenstein and Merrett, 1984; Koudas, 2000). They can be classified (Gaede and Günther, 1998) into approximation, clipping, and mapping methods.

Approximation methods store a hierarchy of approximations of both objects and the space occupied by subsets of objects. Since the space is not partitioned, approximations can overlap. Many approximation methods are proposed, including the Rtree (Guttman, 1984), the $R^{*}$-tree (Beckmann et al., 1990) and the X-tree (Berchtold et al., 1996). Clipping methods, such as the $R^{+}$-tree (Sellis et al., 1987), partition an object into parts so that over- 
lap is avoided. Mapping methods map objects in ndimensional space into a one-dimensional order. The objects are then stored and retrieved using an access method such as a $\mathrm{B}^{+}$-tree (Comer, 1979). Approaches that use mapping include Z-ordering (Orenstein and Merrett, 1984), the Hilbert R-tree (Kamel and Faloutsos, 1994), and the Filter tree (Koudas, 2000).

No n-dimensional to one-dimensional mapping of spatial data exists that preserves all spatial relationships between objects (Gaede and Günther, 1998). A limitation to hierarchical SAMs is their one-dimensional structure. This forces objects in ndimensional space into a one-dimensional ordering, which results in the loss of spatial relationships. This leads to inefficient searching, both within a node and the structure as a whole, because the only option is a linear search of a node in its entirety. Mapping methods do provide a one-dimensional ordering of objects, but they cannot maintain all spatial relationships.

\section{THE 2DR-TREE}

The 2DR-tree is an approximation SAM that uses nodes that are two-dimensional in structure to organize approximations for objects and the space occupied by objects. In every node, an approximation is stored in an appropriate location with respect to all other approximations in the node. Using two-dimensional nodes allows approximations to be placed so that spatial relationships are preserved. We present the 2DR-tree and define key concepts below.

\subsection{Preliminaries}

The 2DR-tree uses a minimum bounding rectangle (MBR) for approximating an object and the space occupied by a subset of objects. An MBR is the minimum extent along both the $x$-axis and the $y$-axis that encompasses an object in a leaf node, and a subset of MBRs in a non-leaf node. The centroid of an MBR are the co-ordinates $(i, j)$ of its centre.

The supported spatial relationships are north, east, south, west, northeast, northwest, southeast, and southwest. A spatial relationship is determined by comparing the centroids between two MBRs. This leads to a smaller set than that in (Papadias et al., 1996), but we feel this simpler technique covers all required cases.

The coverage of an MBR is the total area covered by the rectangle. The coverage of a tree is the total coverage of all MBRs in the tree. The overcoverage of an MBR is the area of the whitespace within a rectangle. The overcoverage of a tree is the total overcov-
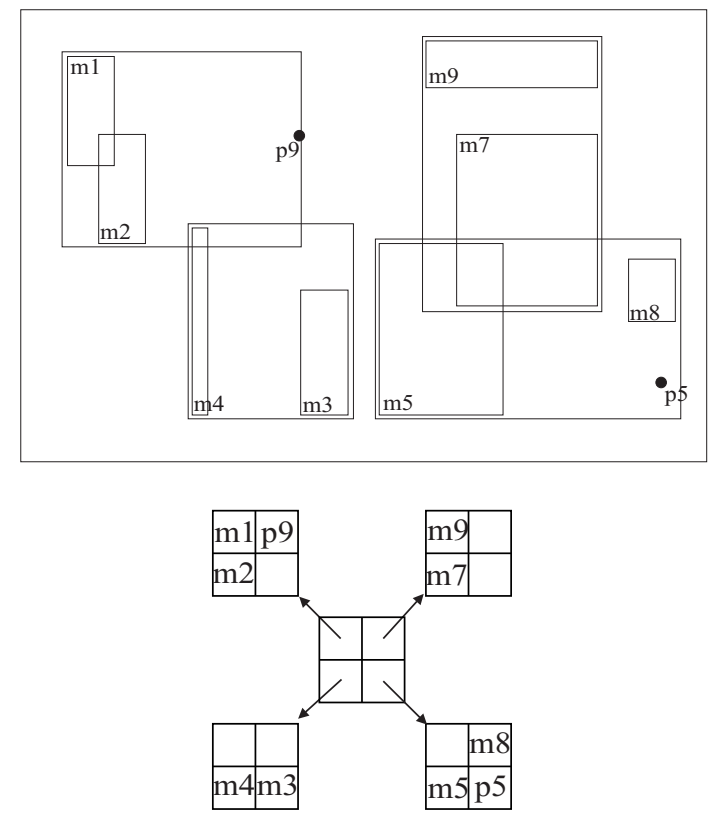

Figure 1: Order $4 * 4$ 2DR-tree.

erage of all MBRs in the tree. The overlap of a tree is the total overlap between all pairs of MBRs.

For each node $N, X$ is the number of indexed locations along the $x$-axis, and $Y$ is the number of indexed locations along the $y$-axis. The order of $N$ is $O=X * Y$. All nodes in a 2DR-tree have the same order. Therefore, the order of a 2DR-tree is the order of its nodes. Each location $(i, j)$ in node $N$ stores:

$$
\left(\operatorname{MBR}_{(i, j)}, p t r_{(i, j)}\right)
$$

where $\operatorname{MBR}_{(i, j)}$ is an MBR and $p t r_{(i, j)}$ is a pointer. In a leaf node, $M B R_{(i, j)}$ encloses an object and $\operatorname{ptr}_{(i, j)}$ references the object on secondary storage. In a nonleaf node, $M B R_{(i, j)}$ encloses all MBRs in the subtree referenced by $\operatorname{ptr}_{(i, j)}$.

A node space is the area of space occupied by a set of objects. This is equal to the MBR that encloses the objects. A node region $(l x, h x, l y, h y)$ is a twodimensional subset of $(x, y)$ index locations in a node. The index values $l x$ and $h x$ are the lower and upper bounds of the node region along the x-axis. The index values $l y$ and $h y$ are the lower and upper bounds of the node region along the $y$-axis.

\subsection{Node Validity}

To employ different searching strategies, the spatial relationships between MBRs in each node must be preserved. For each location $N_{(i, j)}, i=0 \ldots(X-$ 
$1), j=0 \ldots(Y-1)$ in node $N$, if $N_{(i, j)}$ contains $\operatorname{MBR}_{(i, j)}$,

- Location $N_{(k, l)}, k=(i+1) \ldots(X-1), l=0 \ldots j$ contains $\operatorname{MBR}_{(k, l)}$ whose centroid is southeast of the centroid for $M B R_{(i, j)}$,

- Location $N_{(k, l)}, k=(i+1) \ldots(X-1), l=(j+$ 1) ...(Y-1) contains $\operatorname{MBR}_{(k, l)}$ whose centroid is northeast of the centroid for $M B R_{(i, j)}$, and

- Location $N_{(k, l)}, k=0 \ldots i, l=(j+1) \ldots(Y-1)$ contains $\operatorname{MBR}_{(k, l)}$ whose centroid is northwest of the centroid for $M B R_{(i, j)}$.

Figure 1 shows an order $2 * 2$ 2DR-tree that preserves all spatial relationships for the given data set (from (Gaede and Günther, 1998)). Beginning with the leaf node descending from root location $(0,0)$, the centroid for $\mathrm{m} 3$ is located southeast of the centroid so $\mathrm{m} 3$ is located east of $\mathrm{m} 4$ in the node $(\mathrm{m} 4, \mathrm{~m} 3)$. In node (m5,p5,m8), p5 is located southeast of the centroid for $\mathrm{m} 5$, and the centroid for $\mathrm{m} 8$ is located northeast of the centroid for $\mathrm{m} 5$ and northwest of $\mathrm{p} 5$. Therefore, $\mathrm{p} 5$ is stored east of $\mathrm{m} 5$ while $\mathrm{m} 8$ is stored northeast of $\mathrm{m} 5$ and north of p5. Spatial relationships are also maintained in nodes $(\mathrm{m} 7, \mathrm{~m} 9),(\mathrm{m} 2, \mathrm{~m} 1, \mathrm{p} 9)$, and the root node.

\section{2DR-TREE INSERTION}

The 2DR-tree insertion strategy has five stages: 1) search for an appropriate leaf node, 2) search for the appropriate location within the leaf node, 3 ) place the new object with respect to any objects remaining in the node so that spatial relationships are maintained, 4) attempt to put back the objects that were removed so that spatial relationships are maintained - if not possible, then a split is performed, and 5) perform an update of the insertion path. The first four stages are detailed here.

\subsection{Leaf Node Search}

An appropriate leaf node is found for new object $M B R_{n}$ by applying a greedy search to each node on the insertion path. Each chosen node contains an MBR that requires either a minimal or the least area increase necessary to include the new object. The greedy search finds this MBR along a path of decreasing area increases. The most optimal MBR may not be found, but the number involved in the search is reduced. This is because the approximations in the node are now organized, and other search strategies can now be applied.
Beginning at location $(0,0)$, each approximation in location $(i, j)$ on the search path is compared with $(i, j+1),(i+1, j+1)$ and $(i+1, j)$. If the MBR at $(i, j)$ has the smallest area increase, the search terminates in the node and continues in the corresponding subtree. Otherwise, the search continues in the direction that contains an MBR with the smallest area increase. This is repeated at each level of the tree, until a leaf node is reached.

\subsection{Object Location Search}

After a leaf node is found, a location for $M B R_{n}$ is found within the node by performing a recursive partition of the node space $N S$ that corresponds to the leaf node. For each recursive stage, two steps take place. First, the node space is partitioned into two equal subspaces through the dimension with the longest extent. Second, a node region that corresponds to each subspace is identified. The subspace (and corresponding node region) that contains $M B R_{n}$ is selected for further partitioning, while the node region corresponding to the other subspace is removed from the node and will be put back after $M B R_{n}$ is inserted.

Figure 2 shows partitioning for the north and south cases, and how the new object relates to the partition. In Figure $2 \mathrm{a}$, the new object $N$ is located north of the partition. In Figure 2b, $N$ is located south of the partition. In these cases, the north or south subspace respectively is chosen for further partitioning.

Recursive partitioning continues until either one MBR remains in the node $\left(M B R_{r}\right)$, or multiple objects exist and $M B R_{n}$ has some specific spatial relationship with those objects.

\subsection{New Object Placement}

After removing subsets of objects, $M B R_{n}$ is inserted with respect to the remaining object(s) to ensure that all spatial relationships are maintained. We present the cases for insertion relative to one MBR, followed by insertion relative to multiple MBRs.

The cases for insertion $M B R_{n}$ relative to one remaining approximation, $M B R_{r}$ are listed below. $M B R_{r}$ is located at $(i, j)$.

North Insert. $M B R_{n}$ is located northwest of $M B R_{r}$ and the slope of a line between their centroids is less than $-1 . M B R_{n}$ is inserted in location $(i, j+1)$. This is depicted in Figure 3a.

North Swap Insert. $M B R_{n}$ is located southeast of $M B R_{r}$ and the slope of a line between their centroids is less than $-1 . M B R_{n}$ is swapped with $M B R_{r}$ and $M B R_{r}$ is inserted in location $(i, j+1)$. This is depicted in Figure $3 b$. 


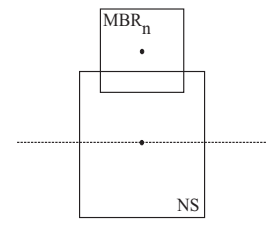

a. North

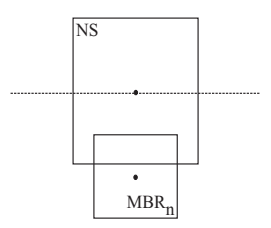

b. South
Figure 2: Partitioning Cases for North and South.

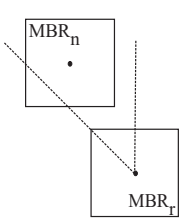

a. North Insert

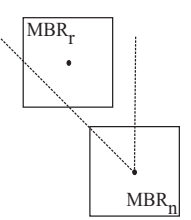

b. North Swap Insert
Figure 3: Single Object Cases for North Inserts.

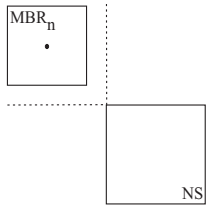

a. North Insert

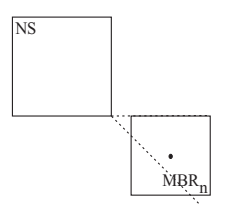

b. East Insert
Figure 4: Multiple Object Cases for North and East.

East Insert. $M B R_{n}$ is located southeast of $M B R_{r}$ and the slope of a line between their centroids is greater than $-1 . M B R_{n}$ is inserted in $(i+1, j)$.

East Swap Insert. $M B R_{n}$ is located northwest of $M B R_{r}$ and the slope of a line between their centroids is greater than $-1 . M B R_{n}$ is swapped with $M B R_{r}$ and $M B R_{r}$ is inserted in $(i+1, j)$.

Northeast Insert. $M B R_{n}$ is located northeast of $M B R_{r} . M B R_{n}$ is inserted in $(i+1, j+1)$.

Northeast Swap Insert. $M B R_{r}$ is located northeast of $M B R_{n} . M B R_{n}$ is swapped with $M B R_{r}$ and $M B R_{r}$ is inserted in $(i+1, j+1)$.

The multiple object cases identify situations where $M B R_{n}$ has certain spatial relationships relative to all remaining approximations in the node. For all cases, the remaining approximations occupy node space $N S$, which corresponds to node region $\left(l x_{r n s}, h x_{r n s}, l y_{r n s}, h y_{r n s}\right)$.

North Insert. $M B R_{n}$ is located northwest of $N S$, and the slope of a line between their centroids is less than -1 . $M B R_{n}$ is inserted in location $\left(l x_{r n s}, h y_{r n s}+1\right)$. This is depicted in Figure 4a.

East Insert. $M B R_{n}$ is located southeast of $N S$, and the slope of a line between their centroids is greater than

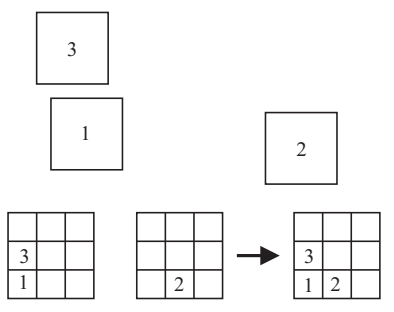

a. East, No Overlap

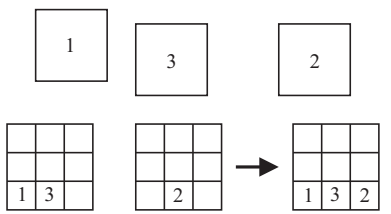

b. East, Overlap

Figure 5: Node Restore for East Cases.

-1. $M B R_{n}$ is inserted in location $\left(h x_{r n s}+1, l y_{r n s}\right)$. This is depicted in Figure $4 \mathrm{~b}$.

Northeast Insert. $M B R_{n}$ is located northeast of $N S$. $M B R_{n}$ is inserted in location $\left(h x_{r n s}+1, h y_{r n s}+1\right)$.

Northeast Swap Insert. $M B R_{n}$ is located southwest of $N S$. The node region is shifted to the northeast (i.e. over one column and up one row), and $M B R_{n}$ is inserted into the original $\left(l x_{r n s}, l y_{r n s}\right)$ location.

\subsection{Restoring the Leaf Node}

After $M B R_{n}$ is inserted, any removed node regions are restored in the reverse order of their removal. After replacing each node region, a validity test is performed. The final result is either a completely restored node, or a set of nodes if a split is required.

Each removed node region has a direction of north, south, east, or west, depending on which side of the partition its corresponding node space was on. This direction is used to restore the node region relative to $\left(l x_{r n s}, h x_{r n s}, l y_{r n s}, h y_{r n s}\right)$. When $M B R_{n}$ is inserted, one or both of the upper node region boundaries $h x_{r n s}$ and $h y_{r n s}$ are increased. When this increase occurs, potential overlap problems arise between the node region $\left(l x_{r n s}, h x_{r n s}, l y_{r n s}, h y_{r n s}\right)$ and some removed node regions, namely the east and north node regions. Therefore, the resulting cases are north, north with overlap, east, east with overlap, south and west.

The east and east with overlap cases are depicted in Figure 5. When east node region $\left(l x_{e}, h x_{e}, l y_{e}, h y_{e}\right)$ does not overlap with $\left(l x_{r n s}, h x_{r n s}, l y_{r n s}, h y_{r n s}\right)$, it is put back into its original location. However, when the east node region $\left(l x_{e}, h x_{e}, l y_{e}, h y_{e}\right)$ does overlaps with 
Table 1: Averages for Varying Object Set Size.

\begin{tabular}{|r|r|r|r|r|r|r|r|}
\hline \#Obj & \#Nodes & Height & Coverage & Overcov & Overlap & \#Seeks/Ins & \#Splits/Ins \\
\hline 100 & 127.97 & 8.10 & $128,906.74$ & $15,801.81$ & $13,070.12$ & 17.82 & 1.04 \\
500 & 654.06 & 12.31 & $1,233,595.22$ & $138,768.90$ & $129,630.80$ & 30.74 & 1.12 \\
1,000 & $1,312.53$ & 14.19 & $3,232,169.34$ & $355,257.04$ & $340,590.69$ & 36.74 & 1.13 \\
2,000 & $2,619,52$ & 16.23 & $8,784,155.25$ & $959,547.75$ & $935,355.26$ & 43.11 & 1.13 \\
4,000 & $5,244.69$ & 18.26 & $23,448,427.79$ & $2,535,300.42$ & $2,494,116.09$ & 49.59 & 1.13 \\
6,000 & $7,867.63$ & 19.47 & $41,887,513.70$ & $4,525,004.07$ & $4,465,319.16$ & 53.43 & 1.13 \\
8,000 & $10,470.28$ & 20.44 & $63,752,968.22$ & $6,862,775.80$ & $6,792,707.51$ & 56.28 & 1.13 \\
10,000 & 13.077 .67 & 21.00 & $88,158,132.24$ & $9,517,985.63$ & $9,432,726.01$ & 58.20 & 1.13 \\
\hline
\end{tabular}

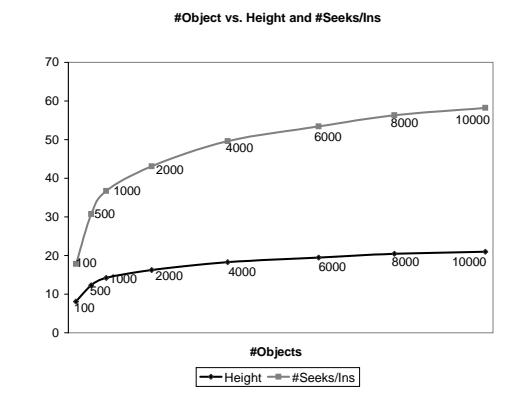

a. \#Objects vs. Height and \#Seeks/Insert

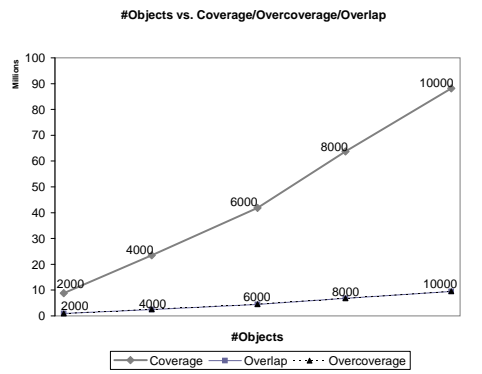

b. \#Objects vs. Coverage/Overlap/Overcoverage

Figure 6: Affect of Object Set Size on Various Parameters.

$\left(l x_{r n s}, h x_{r n s}, l y_{r n s}, h y_{r n s}\right)$, it is shifted one column east from its original position before it is put back. The other cases are handled similarly.

To handle overflow and node invalidity, different splitting strategies are used. One strategy is the reduction split, which takes advantage of the node regions that are removed from the node. Each node region that cannot be put back is assigned to its own node.

\section{PERFORMANCE EVALUATION}

The preliminary performance evaluation observes the behaviour of the 2DR-tree for different object set sizes and distributions. We created object sets that contain between 100 and 10,000 equal-sized squares. With between 51-53\% overlap, each set covers 67$75 \%$ of the space.

Each test run constructs 1000 trees using random sorts of the object set. The tree height, number of nodes, average space utilization, coverage, overcoverage, overlap, average number of disk accesses per insertion, and the average number of splits per insertion are recorded. Each disk access retrieves one node. We assume that each page from secondary storage stores the information for one node, independent of node size. We also assume that with the exception of the root node and new nodes produced from a split, each node is retrieved every time it is read. In the latter case, the MBRs required for updating are generated immediately after creating the node so retrieving new nodes resulting from a split is not required.

We evaluate the insertion algorithm using the following sets of test runs: 1) varying the number of objects between 100 and 10000, using a $5 \times 5$ node size and uniform distribution, 2) varying the data distribution between uniform and exponential, using 500 objects and a $5 \times 5$ node size.

\subsection{Results and Discussion}

Table 1 shows the averages for each run when varying the number of objects inserted into the 2DR-tree. The average number of seeks per insert is less than three times the average tree height in all cases. This includes the number required to find the appropriate leaf node so updating takes the remaining seeks. The average number of seeks for updating is more than one times the average tree height due to the average of approximately one split per insertion occurring. This is significant since splits can be triggered by many situations in the 2DR-tree. The results show that splits are not a significant factor for 2DR-tree insertion.

Figure 6a shows the effect of the number of ob- 
Table 2: Averages for Varying Distribution.

\begin{tabular}{|r|r|r|r|r|r|r|r|}
\hline Distribution & \#Nodes & Height & Coverage & Overcov & Overlap & \#Seeks/Ins & \#Splits/Ins \\
\hline Uniform & 654.06 & 12.31 & $1,233,595.22$ & $138,768.90$ & $129,630.80$ & 30.74 & 1.12 \\
Exponential & 652.63 & 12.31 & $638,430.98$ & $64,157.58$ & $67,130.08$ & 30.71 & 1.10 \\
\hline
\end{tabular}

jects inserted on the tree height, and the average number of disk accesses required for inserting an object. Initially, the tree grows in height quickly but growth slows significantly as more objects are inserted. The same occurs for the number of disk accesses.

Figure $6 \mathrm{~b}$ shows the effect of the number of objects inserted on the coverage, overlap and overcoverage. Results show that the coverage increases linearly as the number of objects increase. In addition, the rate of increase in overlap and overcoverage is significantly lower as the number of objects increase. Coverage includes the object coverage, while overlap and overcoverage are only calculated for non-leaf nodes. One reason for the lower growth in overlap and overcoverage is the ability of the 2DR-tree to "cluster" objects located close together as the number of objects increase, which reduce both the overlap and the wasted space in non-leaf approximations.

Table 2 shows the averages for each run when varying the distribution of the data set. The results show a significant difference in coverage, overcoverage, and overlap. The surprising result is that when indexing exponentially distributed data, the 2DRtree achieves significantly, almost 50\%, lower coverage and overlap, and 54\% lower overcoverage. The height, number of nodes, and space utilization are not a factor in this because they are not significantly different between the data distributions. After many insertions, "chains" that consist of many non-leaf nodes that lead to one node with few objects - possibly one start to appear. An advantage to chains is that outliers are separated from a cluster of objects, which reduces the coverage, overcoverage, and overlap of MBRs.

\section{CONCLUSION}

This paper presents work on the 2DR-tree, which preserves spatial relationships between all objects by using nodes that are the same dimensionality as the object set. This structure supports non-linear search strategies. We present the insertion strategy and some preliminary evaluation results. The results show that the 2DR-tree is ideal for larger objects sets with respect to tree height. The average number of disk accesses and split per insert are reasonable. In addition, it is ideal for a dynamic skewed data set, which achieves lower coverage, overcoverage, and overlap than a dynamic, uniformly distributed data set.

Some research directions include: 1) a performance evaluation versus other proposed SAMs; 2) improving the average space utilization, which is very low; 3) developing an algorithm for bottom-up tree construction applicable to static data sets; 4) extending the 2DR-tree for three dimensions.

\section{REFERENCES}

Beckmann, N., Kriegel, H.-P., Schneider, R., and Seeger, B. (1990). The $\mathrm{R}^{*}$-tree: an efficient and robust access method for points and rectangles. In Proceedings of the ACM SIGMOD International Conference on Management of Data, pages 322-31.

Berchtold, S., Keim, D., and Kriegel, H.-P. (1996). The Xtree: An index structure for high-dimensional data. In Proceedings of the 22nd International Conference on Very Large Data Bases, pages 28-39.

Comer, D. (1979). The ubiquitous B-tree. ACM Computing Surveys, 11:121-37.

Gaede, V. and Günther, O. (1998). Multidimensional access methods. ACM Computing Surveys, 30:170-231.

Guttman, A. (1984). R-trees: a dynamic index structure for spatial searching. In Proceedings of the ACM SIGMOD International Conference on Management of Data, pages 47-57.

Kamel, I. and Faloutsos, C. (1994). Hilbert R-tree: an improved r-tree using fractals. In Proceedings of the 20th International Conference on Very Large Data Bases, pages 500-9.

Koudas, N. (2000). Indexing support for spatial joins. Data and Knowledge Engineering, 34:99-124.

Orenstein, J. and Merrett, T. (1984). A class of data structures for associative searching. In Proceedings of the Third ACM SIGACT-SIGMOD Symposium on Principles of Database Systems, pages 181-90.

Papadias, D., Egenhofer, M., and Sharma, J. (1996). Hierarchical reasoning about direction relations. In Proceedings of the 4th ACM-GIS.

Samet, H. (1990). The design and analysis of spatial data structures. Addison-Wesley.

Sellis, T., Roussopoulos, N., and Faloutsos, C. (1987). The $\mathrm{R}^{+}$-tree: a dynamic index for multi-dimensional objects. In Proceedings of the 13th International Conference on Very Large Data Bases.

Shekhar, S. and Chawla, S. (2003). Spatial databases: a tour. Prentice Hall. 